\title{
Hypersensitivity of BRCA1 Heterozygote Lymphoblastoid Cells to Gamma Radiation and PARP Inhibitors
}

\author{
Bourton EC ${ }^{1}$, Foster HA${ }^{1}$, Plowman $\mathrm{PN}^{2}$, Harvey $\mathrm{AJ}^{1}$ and Parris $\mathrm{CN}^{1 *}$ \\ ${ }^{1}$ Brunel Institute of Cancer Genetics and Pharmacogenomics, Division of Biosciences, School of Health Sciences and Social Care, Brunel \\ University, Uxbridge, Middlesex UB8 3PH, United Kingdom \\ ${ }^{2}$ Department of Radiotherapy, St Bartholomew's Hospital, West Smithfield, London EC1A 7BE, United Kingdom
}

\begin{abstract}
PARP inhibitors can be used to induce synthetic lethality in cells with bi-allelic BRCA1 and BRCA2 mutations. However the effect of PARP inhibitors in combination with radiation on cells with mono-allelic mutations of $B R C A 1$ and $B R C A 2$ is unknown. We have examined the cell survival response of lymphoblastoid cells derived from normal individuals and those derived from carriers of $B R C A 1$ and $B R C A 2$ mutations, following exposure to ionising radiation and the PARP inhibitor Olaparib.

Two lymphoblastoid cell lines from normal individuals and three with mono-allelic mutations in BRCA1 and $B R C A 2$ were exposed to increasing doses of gamma radiation either alone or in combination with $5 \mu \mathrm{M}$ Olaparib. Cell survival was measured using the MTT assay.

Exposure to increasing doses of gamma radiation caused a reduction in cell survival of all cell types. The combined exposure to gamma radiation and $5 \mu \mathrm{M}$ Olaparib did not enhance cell kill in normal or BRCA2 heterozygote lymphoblastoid cells but significantly enhanced cell kill in cells derived from $B R C A 1$ carriers $(P=0.02)$. The treatment of cancer patients carrying mutations in the BRCA1 gene with radiotherapy and the PARP inhibitor Olaparib may significantly enhance radiation induced normal tissue toxicity in these patients.
\end{abstract}

Keywords: $B R C A 1$; BRCA2; Heterozygote; Radiosensitivity; PARP inhibitor

\section{Introduction}

BRCA1 (breast cancer susceptibility gene 1) and BRCA2 are important 'caretaker' tumour suppressor genes involved in many cellular processes. In particular, the products of both genes are central to the mechanisms of DNA repair and transcriptional regulation in response to DNA damage [1]. BRCA1 and BRCA2 are critical in the repair of DNA double strand breaks (DSB) which are the predominant form of cellular injury caused by exposure to ionising radiation [2]. Human cells utilise two principal mechanisms to repair DNA DSB. In non-cycling and cells in early G1, non homologous end joining (NHEJ) is predominant. Here DNA DSB are ligated together in an error prone manner and central to this process is the DNA dependent protein kinase (DNA-PK) and its associated proteins Ku70 and Ku80 together with the end processing enzyme artemis. Finally ligation of broken ends is mediated by the XRCC4-ligase IV complex [3].

Alternatively, in cycling cells in the later phases of the cell cycle, DSB repair is mediated by the process of homologous recombination (HR), whereby the repair of the DSB is achieved through exchange of genetic material from the homologous chromosome or sister chromatid during DNA replication [4].

The BRCA1 protein, by interactions with tumour suppressor proteins (such as P53), cell cycle regulators and DNA repair proteins fulfils diverse roles in a variety of DNA repair pathways including NHEJ, HR and single strand annealing (SSA). BRCA1 deficient cells are characterised by severe patterns of chromosome aberrations and extreme genomic instability [5]. BRCA2 on the other hand is primarily involved in the process of HR. BRCA2 has been shown to bind to the Rad51 recombinase protein which causes strand exchange during the process of DSB repair by HR. BRCA2-deficient cells are ineffective in recruiting Rad51 to DSB sites and are therefore defective in HR [6].
Mutations in both the BRCA1 and BRCA2 genes have been demonstrated to be central to the development of a number of types of cancer, in particular breast cancer. While the majority of breast cancers are sporadic, germline mutations of the BRCA1 and BRCA2 genes are associated with some $5-10 \%$ of breast cancers where the disease is characterised by early onset (usually before the age of 40). Also, individuals with such mutations also have an increased susceptibility to ovarian, pancreatic, prostatic and male breast cancers [1,7].

A fundamental treatment method for most locally advanced cancers, including breast cancer, is clinical fractionated radiotherapy, used either as a single modality or in combination with surgery and/or chemotherapy [8]. The effectiveness of cancer treatment is frequently limited by the development of side effects in the form of normal tissue toxicity (NTT). NTT can occur both during treatment (acute response) and post-treatment (late response) in a variety of tissues and is experienced to a greater or lesser extent by $15-20 \%$ of all radiotherapy patients [8]. Normal tissue reactions have been appreciated for well over half a century of clinical radiotherapy practice and have been standardised into established scales of NTT for all tissue types $[9,10]$. In addition to the observed standardised scale of NTT, a small

*Corresponding author: Christopher N Parris, Brunel Institute of Cance Genetics and Pharmacogenomics, Division of Biosciences, School of Health Sciences and Social Care, Brunel University, Uxbridge, Middlesex, UB8 $3 \mathrm{PH}$, United Kingdom, Tel: +44 (0)1895 266293; Fax: +44 (0)1895 269873; E-mail: christopher.parris@brunel.ac.uk

Received April 29, 2013; Accepted June 13, 2013; Published June 17, 2013

Citation: Bourton EC, Foster HA, Plowman PN, Harvey AJ, Parris CN (2013) Hypersensitivity of BRCA1 Heterozygote Lymphoblastoid Cells to Gamma Radiation and PARP Inhibitors. J Genet Syndr Gene Ther 4: 146. doi:10.4172/21577412.1000146

Copyright: (C) 2013 Bourton EC, et al. This is an open-access article distributed under the terms of the Creative Commons Attribution License, which permits unrestricted use, distribution, and reproduction in any medium, provided the original author and source are credited. 
minority of individuals manifest toxicity reactions that fall outside such classifications and these individuals are referred to as 'overreactors'. Individuals with inherited genetic defects in the repair of DNA DSB are over-reactors. Classical examples of radiotherapy overreactors include individuals with Ataxia Telangiectasia (A-T), where an inactivating mutation in the ATM gene results in aberrant cell cycle control, defective DNA DSB repair and extreme clinical and cellular hypersensitivity to ionising radiation [11]. In addition, we have also demonstrated that an inactivating mutation in the DNA-PKcs gene (which is central to NHEJ DSB repair), resulted in extreme cellular and clinical radiosensitivity eventually leading to the patient's death as a result of radiotherapy-induced neurological trauma [12].

Due to the fact that the BRCA1 and BRCA2 proteins are central to proficient DNA DSB repair by either NHEJ or HR, it is possible that individuals carrying mutations in either of these genes might represent a cohort of patients in which radiotherapy-induced NTT might be over and above the normal range expected. Indeed cellular clonogenic assays in cells derived from $B R C A 1$ and $B R C A 2$ heterozygotes reveal elevated radiosensitivity and chromosome aberrations when compared to cells derived from normal individuals, which is suggestive of a haplo-insufficient phenotype [13]. However, clinical evidence suggests that heterozygotes for both BRCA1 and BRCA2 do not experience abnormally severe NTT [14].

More recently however a novel class of drugs targeted to inhibit poly(ADP-ribose) polymerase (PARP) are being investigated for the treatment of patients with BRCA1 and BRCA2-negative tumours. Several members of the PARP enzyme family have roles in DNA repair. However, PARP-1 accounts for approximately $90 \%$ of cellular PARP activity [15]. PARP-1 is essential for the repair of DNA single strand breaks primarily through the mechanism of base excision repair, and inhibition of PARP-1 causes the creation of a DNA DSB at the site of damage during DNA replication [16]. The first PARP inhibitors were nicotinamide analogues such as the benzamides. One such compound 3-Aminobenzamide was initially used in the clinical setting in combination with cytotoxic chemotherapeutic drugs, rather than radiotherapy. Later studies on cell lines from head and neck cancer, and from lymphoma, have shown that the PARP inhibitor AZD-2281 (Olaparib), when used with ionising radiation, produced a synergistic effect [17]. While the use of PARP inhibitors for treating patients with $B R C A 1$ and $B R C A 2$ deficient tumours might be an effective approach in the clinical management of such cancers, the combination of both radiotherapy and PARP inhibition may represent a mechanism whereby the effectiveness of radiotherapy may be enhanced. The corollary of this approach however, is that patients treated with such a combination may experience elevated normal tissue toxicity in cells heterozygous for either $B R C A 1$ or $B R C A 2$ mutations. To address this question we have examined the cellular sensitivity of $B R C A 1$ and BRCA2 heterozygous cells to radiation in the presence and absence of the PARP-1 inhibitor Olaparib. We demonstrate that B-lymphoblastoid cells heterozygous for $B R C A 1$ show elevated cellular sensitivity to radiation in the presence of Olaparib when compared to heterozygous BRCA2 B-lymphoblastoids and those from normal individuals. We caution that heterozygous $B R C A 1$ patients treated with radiotherapy and PARP inhibitors may experience abnormally high levels of normal tissue toxicity.

\section{Materials and Methods}

\section{Cell lines}

Human B lymphocytes which had been immortalised using the
Epstein-Barr virus were purchased from Coriell Cell Repositories (Camden, New Jersey, USA). Details of the cell lines are shown in Table 1. Cell lines "BRCA1" and "BRCA2" were heterozygotes for mutations in the BRCA1 or BRCA2 genes, respectively.

\section{Cell culture}

The cell lines were initially established in T25 cell culture flasks (PAA Laboratories Limited, Yeovil, Somerset, UK) in RPMI 1640 culture medium (Sigma-Aldrich, Poole, Dorset, UK) supplemented with $10 \%$ foetal bovine serum, $2.0 \mathrm{mM} \mathrm{L}$-Glutamine and $100 \mathrm{Uml}^{-1}$ Penicillin and Streptomycin (PAA). Upon reaching 75-80\% confluence, cell lines were transferred to T75 flasks for further culture. Cells were routinely incubated at $37^{\circ} \mathrm{C}$ in a humidified atmosphere of $5 \% \mathrm{CO}_{2}$ in air.

Values for cell concentration (number of cells per $\mathrm{ml}$ cell suspension) and viability (\% live cells/dead cells) were determined as required using a "Countess" ${ }^{\text {ra }}$ " automated cell counter based upon the method of trypan blue exclusion (Invitrogen, Paisley, Renfrewshire, UK). Cell cultures were used over a restricted range of ten passages, during which cell viability was not less than $80 \%$.

\section{MTT assay}

Due to the fact that the lymphoblastoid cells used in the study did not form colonies in a standard clonogenic assay, the extensively used and widely accepted MTT assay was conducted as described previously $[18,19]$ using an MTT assay kit (Merck-Millipore Ltd., Watford, UK).

In brief, the 3-(4,5-dimethylthiazol-2-yl)-2,5-diphenyl tetrazolium bromide (MTT) reagent $(50 \mathrm{mg}$ ) was dissolved in $10 \mathrm{ml}$ PBS (pH 7.4) and sterilised by filtration. Ten microlitres of this solution was added to each well containing cell suspension on the 96-well microplates, and the contents mixed by gentle agitation of the plate, followed by incubation for 3 hours (incubation conditions detailed previously). Colour development was achieved by the addition of $10 \mu \mathrm{L}$ propan-2ol with $0.04 \mathrm{~N} \mathrm{HCl}$ and well contents were mixed by retro-pipetting. A "BP800" microplate reader (Biohit PLC, Helsinki, Finland) was used to obtain absorbance values of each well with a test wavelength of $562 \mathrm{~nm}$ and a reference wavelength of $630 \mathrm{~nm}$.

A cell dilution assay was performed to determine the optimum cell concentration and volume of cell suspension for use in the MTT assay. This was to ensure that the optical density achieved in this assay fell within the linear range. It was determined that the optimum values were 750000 cells per $\mathrm{ml}$ in a volume of $100 \mu \mathrm{L}$ cell suspension per plate well giving 75000 cells per plate well.

\section{Exposure to radiation}

The stock cell suspension of each cell line was divided in half and each diluted with either complete medium only or a solution of PARP inhibitor in complete medium to create two separate cell suspensions of concentration 750000 cells per ml. Each suspension was divided into five aliquots in $15 \mathrm{ml}$ centrifuge tubes. One aliquot from each set was designated as an un-irradiated control; the remaining four aliquots were irradiated with 2, 4, 6 or 8-Gy gamma radiation from a ${ }^{60}$ Cobalt source (Puridec Technologies, Oxfordshire, UK) sited at a distance of $25 \mathrm{~cm}$ with a dose rate between 1.0 and $1.2 \mathrm{~Gy}$ per minute.

The un-irradiated and 2, 4, 6, and 8-Gy irradiated cells from both the untreated and PARP-inhibitor-exposed (Olaparib) cells were seeded using a multi-channel pipette into 96-well microplates in quadruplicate (four identical wells). Separate plates were prepared such 
Citation: Bourton EC, Foster HA, Plowman PN, Harvey AJ, Parris CN (2013) Hypersensitivity of BRCA1 Heterozygote Lymphoblastoid Cells to Gamma Radiation and PARP Inhibitors. J Genet Syndr Gene Ther 4: 146. doi:10.4172/2157-7412.1000146

that independent MTT assays could be performed on each cell line at 3, 5 and 7 days post-irradiation. The cells were incubated for 3, 5 or 7 days (incubation conditions detailed previously). Although survival was estimated at days 3, 5 and 7, the data shown in the results section are derived from a 7 day period following radiation exposure. This was chosen to allow maximum time for cell recovery and thus giving an accurate reflection of cytoxicity caused by the radiation and Olaparib exposure.

\section{Exposure to the PARP inhibitor Olaparib}

To determine the highest non-cytotoxic concentration of Olaparib (LC Laboratories Inc, Woburn, Massachusetts, USA) cells were exposed to increasing concentrations of the drug in the range of 1.0 $\mu \mathrm{M}$ to $1000.0 \mu \mathrm{M}\left(\log _{10}\right.$ scale). The cells were incubated in the drug for 3, 5 and 7 days and survival was determined using the MTT assay. The appropriate concentration of Olaparib was found to be $5.0 \mu \mathrm{M}$ following a 7 day exposure; this was the maximum concentration of Olaparib that did not cause significant cell death

\section{Statistical analysis}

To compare differences in cell survival after exposure to radiation either alone or in combination with Olaparib, a Student's unpaired $t$-Test was used by comparing the survival of cells after exposure to 2 Gy gamma radiation (SF2 values).

\section{Results}

\section{Olaparib sensitivity of lymphoblastoid cell lines}

The response of the lymphoblastoid cells to increasing concentrations of Olaparib is shown in Figure 1. It can be observed that increasing the concentration of Olaparib results in a proportionate decrease in cell survival of the normal lymphoblastoid cells and the BRCA1 and BRCA2 heterozygote cells. There were no marked/ significant differences in response between the three classes of cell line. For future experiments it was decided that a concentration of Olaparib of $5 \mu \mathrm{M}$ would be used as a dose which resulted in minimal toxicity to all cell lines.

\section{Response of lymphoblastoid cell lines to radiation $+/-$ Olaparib}

The response of the normal lymphoblastoid cell lines GM00893 and GM05423 to 0, 2, 4, 6 and 8 Gy gamma radiation is shown in Figures $2 \mathrm{~A}$ and $2 \mathrm{~B}$ respectively. For each cell line there is a reduction in cell survival with increasing dose of gamma radiation. In the GM00893 cell line, cell kill is slightly (but not significantly) enhanced in the presence of $5 \mu \mathrm{M}$ Olaparib (Figure 2A). However, for the GM05423 cell lines the exposure of cells to Olaparib does not enhance cell kill following radiation exposure (Figure $2 \mathrm{~B}$ ). Figure $2 \mathrm{C}$ depicts the mean survival of the two cell lines plotted as a single graph.

Three lymphoblastoid cell lines harbouring mono-allelic mutations in the BRCA1 gene were examined (GM13705, GM14090 and GM16105). The data are shown in Figures 3A-3D. For the GM13705 lymphoblastoid cells, exposure to increasing doses of gamma radiation reduces cell survival and although cell kill is slightly increased by the presence of $5 \mu \mathrm{M}$ Olaparib, this is not a significant enhancement (Figure 3A). For the GM14090 and GM16105 cells, we observed a significant potentiation of cell kill when the cells were irradiated in the presence of $5 \mu \mathrm{M}$ Olaparib (Figure 3B and 3C). Figure 3D shows the response of all cells to radiation when plotted as a single graph. The enhancement of radiation induced cell kill by Olaparib is clearly shown and is a significant increase in cell kill $(p=0.02)$.

Also the response of three B-lymphoblastoid cell lines with monoallelic mutations in the BRCA2 gene to increasing doses of gamma radiation in the presence and absence of Olaparib, was investigated and the data are shown in Figures 4A-4D. Figure 4A shows the response of the GM14170 cells and the data indicate that increasing doses of gamma radiation only marginally reduces cell survival in this line and moreover, this response is not enhanced by the presence of Olaparib. Figure 4B shows the response of the GM14622 cells to gamma radiation. Interestingly the cells are significantly more sensitive to the lethal effects of gamma radiation than the GM14170 cells shown in Figure 4A and moreover, the cytotoxic effect of radiation exposure is enhanced by the presence of Olaparib. In Figure 4C, the response of the GM14805 cells is shown and the data indicates that Olaparib does not enhance the cytotoxicity of radiation at any dose examined. In Figure $4 \mathrm{D}$, the data derived from the three cell lines is plotted as a single survival curve with and without Olaparib exposure. These data show that overall there is no significant difference in cell survival of BRCA2 mutation carrier cells in the presence or absence of Olaparib.

To determine if there were significant differences in the response of normal lymphoblastoid cells and those derived from BRCA1 and BRCA2 mono-allelic cells, the survival of cells following exposure to 2 Gy gamma radiation (SF2 values) was compared and the data are shown in Table 2. Comparison of survival at 2 Gy is particularly important because 2 Gy represents a dose of irradiation that induces potentially lethal damage (PLD). PLD repair can be affected by number parameters such as defects in DNA repair or, in this study, the induction of synthetic lethality by PARP inhibition. Two Gray irradiation is also an important clinical dose due to the fact that most fractionated radiotherapy treatments are given in 2 Gy fractions. By comparing the SF2 survival levels of normal and mono-allelic BRCA1

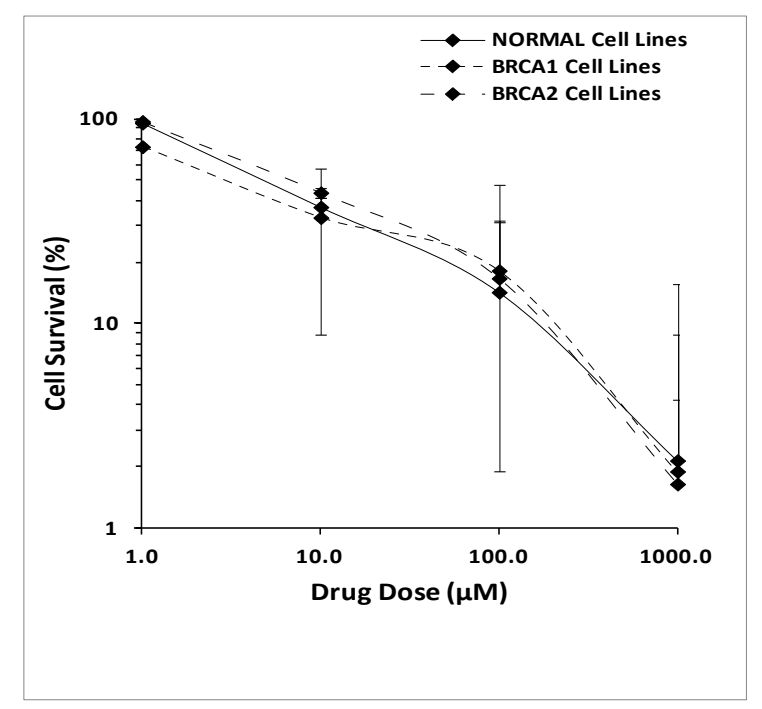

Figure 1: shows the survival of normal lymphoblastoid cells and those derived from individuals with mono-allelic mutations in the BRCA1 and $B R C A 2$ genes. Two normal cell lines are combined to produce the survival curve and three are combined to produce the data for the BRCA1 and BRCA2 least three independent experiments. Due to the logarithmic scale used for the Olaparib concentrations ( $x$ axis) survival at zero dose is not shown. However, survival was $100 \%$. A concentration of $5 \mu \mathrm{M}$ Olaparib was chosen for all future experiments as representing concentration with minimal toxicity to all cell lines. 
Citation: Bourton EC, Foster HA, Plowman PN, Harvey AJ, Parris CN (2013) Hypersensitivity of BRCA1 Heterozygote Lymphoblastoid Cells to Gamma Radiation and PARP Inhibitors. J Genet Syndr Gene Ther 4: 146. doi:10.4172/2157-7412.1000146
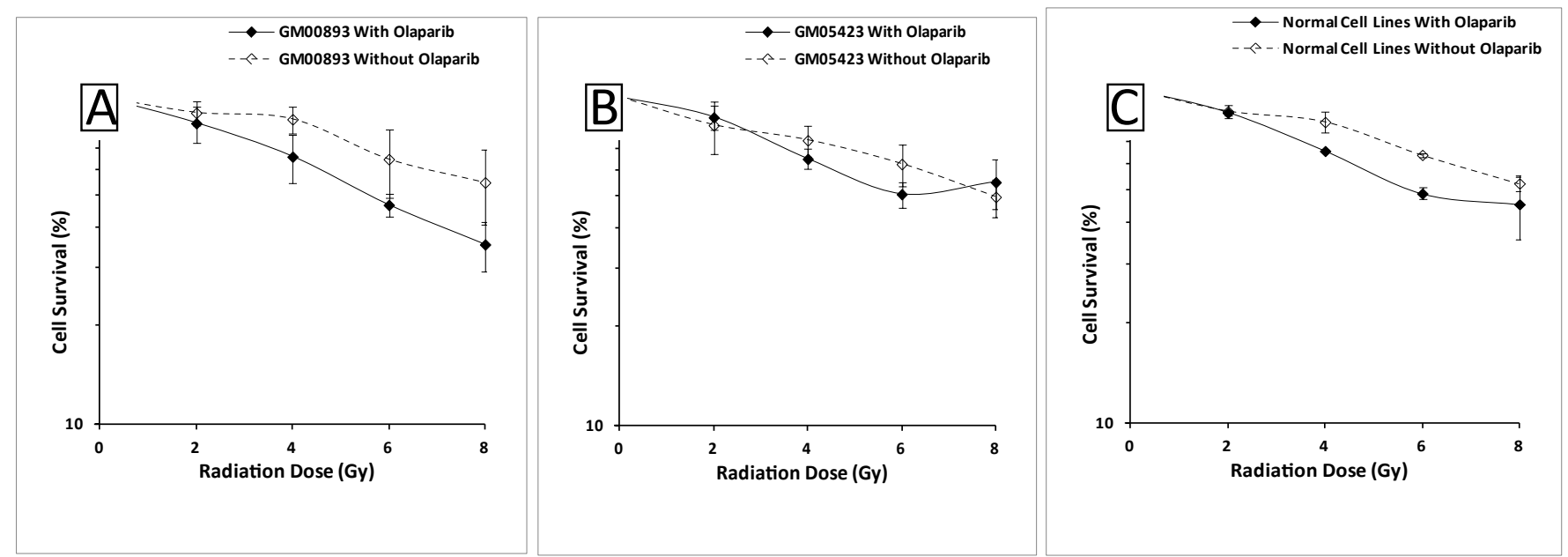

Figures 2: A-C show the response of the two normal lymphoblastoid cell lines to increasing doses of gamma radiation in the presence and absence of $5 \mu \mathrm{M}$ Olaparib. The GM00893 cell line is shown in Figure $2 \mathrm{~A}$ and the GM05432 cell line is shown in figure $2 \mathrm{~B}$. Figure $2 \mathrm{C}$ combines two normal cell lines into a single survival curve. Broken lines show the response of the cells to gamma radiation alone while the unbroken line shows the response to gamma radiation in the presence of Olaparib. Error bars represent standard error of the mean of at least three independent experiments.
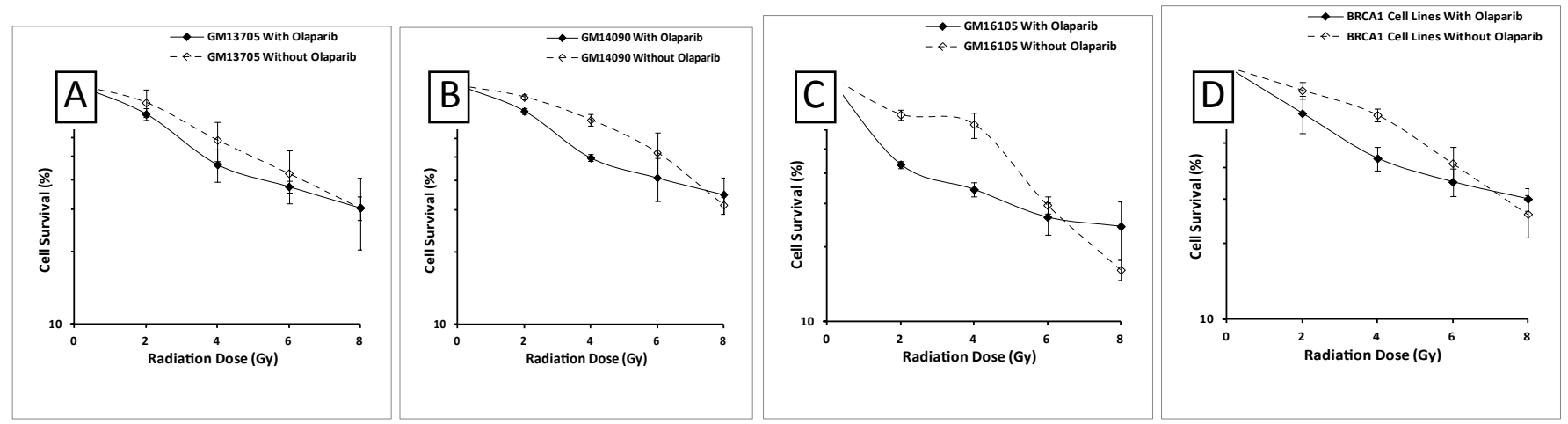

Figures 3: A-D show the response of the three lymphoblastoid cell lines with mono-allelic mutations in the $B R C A 1$ gene to increasing doses of gamma radiation in the presence and absence of $5 \mu \mathrm{M}$ Olaparib. The GM13705 cells line is shown in Figure 3A, GM14090 in Figure 3B and GM16105 in Figure 3C. Figure 3D combines the three responses into a survival curve. Broken lines show the response of the cells to gamma radiation alone while the unbroken line shows the response to gamma radiation in the presence of Olaparib. Error bars represent standard error of the mean of at least three independent experiments.
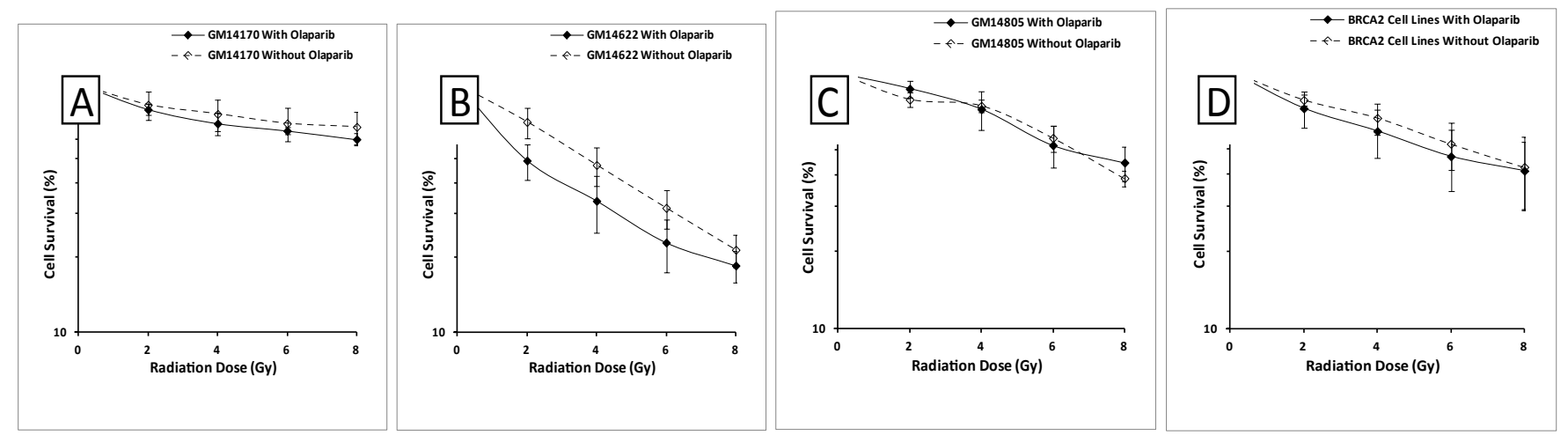

Figures 4: A-D show the response of the three lymphoblastoid cell lines with mono-allelic mutations in the $B R C A 2$ gene to increasing doses of gamma radiation in the presence and absence of $5 \mu \mathrm{M}$ Olaparib. The GM14170 cells line is shown in Figure 4A, GM14622 in Figure 4B and GM14805 in Figure 4C. Figure 4D combines the three responses into a survival curve. Broken lines show the response of the cells to gamma radiation alone while the unbroken line shows the response to gamma radiation in the presence of Olaparib. Error bars represent standard error of the mean of at least three independent experiments. 
Citation: Bourton EC, Foster HA, Plowman PN, Harvey AJ, Parris CN (2013) Hypersensitivity of BRCA1 Heterozygote Lymphoblastoid Cells to Gamma Radiation and PARP Inhibitors. J Genet Syndr Gene Ther 4: 146. doi:10.4172/2157-7412.1000146

Page 5 of 6

cells following exposure increasing doses of gamma radiation, using Student's unpaired T-test, it was seen that there was no significant difference in survival $(p=0.48)$. Similarly, by comparing the normal lymphoblastoid and mono-allelic BRCA2 cells, there was no significant difference in survival after exposure to increasing doses of gamma radiation $(p=0.227)$. Also for the normal and BRCA2 mono-allelic cells, the survival after radiation exposure was not enhanced with $5 \mu \mathrm{m}$ Olaparib ( $p=0.934$ for normal cells plus and minus Olaparib; $p=0.705$ for BRCA2 cells plus and minus Olaparib). However, when BRCA1 mono-allelic cells were irradiated in the presence of Olaparib, the cytotoxicity of gamma radiation was significantly enhanced when the SF2 values were compared $(p=0.02)$.

\section{Discussion}

The increasing use of PARP inhibitors in the clinical management of tumours with bi-allelic mutations in the BRCA1 and BRCA2 genes is a novel mechanism of specifically inducing cell kill in the tumour cells while theoretically avoiding normal cell damage. The induction of cell kill by PARP inhibitors in BRCA1 and BRCA2 cells relies on the mechanism of synthetic lethality by inducing two conditions in cells that individually would not affect cell survival but together can significantly alter a cellular phenotype. In the case of BRCA1 and $B R C A 2$ defective cells, the inhibition of PARP-1 and the resultant suppression of base excision repair results in the conversion of DNA single strand break to DNA DSB which results in the collapse of DNA replication forks leading to cell death. There is significant experimental and clinical evidence that PARP inhibition results in increased tumour cell death [20]. In addition, clinical and experimental evidence suggests that PARP inhibition does not affect the survival of cells with monoallelic mutations of BRCA1 and BRCA2. However, the combination of the use of PARP inhibitors with other forms of cytotoxic cancer treatment may cause significant toxicity in cells with a mono-allelic mutation of either of these genes.

In this study we conducted an in vitro study to determine if cells heterozygous for $B R C A 1$ and $B R C A 2$ mutation would display elevated sensitivity to radiation when treated in the presence of the PARP-1 inhibitor Olaparib. While lymphoblastoid cells from normal individuals and those carrying a mono-allelic mutation in the BRCA2 gene did not display elevated radiosensitivity, the three lymphoblastoid cell lines with mono-allelic mutations in the BRCA1 gene exhibited a significant reduction in cell survival after radiation exposure in combination with PARP-1 inhibition by Olaparib.

Individual differences in cell line sensitivity may in part account for the observed differences in sensitivity between the groups of cell lines. However when the individual cell line data is combined and plotted as a single graph as in Figure 3D and 4D it was clear that there were significant differences between BRCA1 and BRCA2 mutation carriers. (Response to reviewer 1 point 1 and 2). Therefore these observations suggest that a haplo-insufficient phenotype can be exposed in BRCA1 carrier cells when the combination PARP inhibition and radiation is used to kill cells. As a result this observation may have significant clinical implications.

Since the use of radiotherapy for the treatment of cancer, a significant limitation in its use for the treatment of all cancer types is the phenomenon of normal tissue toxicity. NTT is experienced by some $15-20 \%$ of all radiotherapy patients to a greater or lesser degree and moreover, normal fractionated clinical radiotherapy has the potential to be fatal in those individuals with inherited defects in DNA DSB repair mechanisms such as A-T [11]. Thus the clinical application of a strategy to inhibit a DNA repair process prior to radiotherapy (in this case the inhibition of base excision repair by PARP-1 suppression) in patients carrying BRCA1 mutations may increase the number of patients experiencing NTT.

The reasons as to why this enhancement of sensitivity is observed only in the BRCA1 heterozygote cells is interesting and may be due to a number of factors. Firstly the specific nature of the mono-allelic mutation in the BRCA1 and BRCA2 cell lines may confer differential levels of functional protein in each cell line thus influencing the response to radiation and the PARP-1 inhibitor Olaparib. By examining

\begin{tabular}{|l|l|l|}
\hline Cell Line & Type & Details of $B$ RCA Gene Mutation \\
\hline GM00893 & Normal & None \\
\hline GM05423 & Normal & None \\
\hline GM13705 & BRCA1 & 4-base pair deletion in exon 11 = truncated protein. \\
\hline GM14090 & BRCA1 & 2-base pair deletion in exon 3 = truncated protein. \\
\hline GM16105 & BRCA1 & Base substitution in intron 8. \\
\hline GM14170 & BRCA2 & 1-base pair deletion in exon 11 = frameshift mutation. \\
\hline GM14622 & BRCA2 & 2-base pair deletion in exon 11 = frameshift mutation = truncated protein. \\
\hline GM14805 & BRCA2 & Base substitution in exon 7 = nonsense mutation. \\
\hline
\end{tabular}

Table 1 provides a description of the B-Lymphoblastoid cell lines used in the study together with the information on the mono-allelic mutation in the $B R C A 1$ and $B R C A 2$ heterozygous cell lines

Table 1: Description of B-Lymphoblastoid cell lines used in study.

\begin{tabular}{|c|c|c|c|c|c|c|c|c|c|c|c|c|c|c|c|c|}
\hline & \multicolumn{16}{|c|}{ CELL LINE } \\
\hline & \multicolumn{4}{|c|}{ NORMAL } & \multicolumn{6}{|c|}{ BRCA1 } & \multicolumn{6}{|c|}{ BRCA2 } \\
\hline & \multicolumn{2}{|c|}{ GM00893 } & \multicolumn{2}{|c|}{ GM05423 } & \multicolumn{2}{|c|}{ GM13705 } & \multicolumn{2}{|c|}{ GM14090 } & \multicolumn{2}{|c|}{ GM16105 } & \multicolumn{2}{|c|}{ GM14170 } & \multicolumn{2}{|c|}{ GM14622 } & \multicolumn{2}{|c|}{ GM14805 } \\
\hline Olaparib & + & - & + & - & + & - & + & - & + & - & + & - & + & - & + & - \\
\hline SF2 Mean (\%) & 83.2 & 89.5 & 86.9 & 82.3 & 75.3 & 84.1 & 78.1 & 89.4 & 43.5 & 69.3 & 79.1 & 82.7 & 49.3 & 70.5 & 87.1 & 79 \\
\hline SF2 Standard Error & 10.6 & 7.5 & 7.1 & 15 & 7.3 & 8.2 & 2.2 & 2.2 & 1.4 & 3.1 & 4.0 & 11.0 & 8.2 & 10.0 & 6.5 & 5.1 \\
\hline
\end{tabular}

Table 2 shows the surviving fraction following exposure to 2 Gy gamma radiation (SF2). For each cell line the data are derived from a minimum of three independent experiments. The means SF2 for each cell lines with or without incubation with $5 \mu \mathrm{M}$ Olaparib is shown together with the standard error of the mean SF2. Statistical analysis using a Student's unpaired T-test show that there are no significant differences in the SF2 values when the normal lymphoblastoid cells are compared with either the $B R C A 1$ and BRCA2 heterozygous cells. However, BRCA1 heterozygous cells are significantly more sensitive to ionising radiation in the presence of the PARP-1 inhibitor Olaparib $(P=0.02)$

Table 2: Surviving fraction at 2 Gray (SF2) of each cell line irradiated with gamma radiation. 
Citation: Bourton EC, Foster HA, Plowman PN, Harvey AJ, Parris CN (2013) Hypersensitivity of BRCA1 Heterozygote Lymphoblastoid Cells to Gamma Radiation and PARP Inhibitors. J Genet Syndr Gene Ther 4: 146. doi:10.4172/2157-7412.1000146

the mutation in each of the three BRCA1 and three BRCA2 cell lines in Table 1 , it shows that of the three BRCA1 cell lines two contain deletions resulting in truncated proteins and one has an intron 8 mutation presumably affecting RNA splicing. Thus it is unlikely that these alleles are capable of producing a functional protein. A similar analysis with the BRCA2 cell lines reveal two with deleterious frameshift mutations and one with a mutation leading to the introduction of a nonsense codon. Again it is unlikely that these alleles are functional. Therefore it is probable that similar levels of protein are being produced in the cell lines and other factors are likely to be responsible for this interesting phenotype.

Another factor which may underpin the specific $B R C A 1$ phenotype observed in this study is related to the differential function of the BRCA1 and BRCA2 proteins. BRCA1 has an important role in NHEJ, HR, SSA and is known to interact with tumour suppressor genes (such as P53) and other cell cycle regulators. Therefore a mono-allelic mutation of BRCA1 might lead to haplo-insufficient phenotypes in DNA repair when PARP-1 is inhibited increasing the number of DNA double strand breaks. However, the effect of PARP-1 inhibition on a BRCA2 heterozygote cell might be more tolerable. This is because the role of BRCA2 protein is specific to the process of $\mathrm{HR}$ where it is involved in loading Rad51 recombinase at the sites of DNA DSB. Therefore, DNA repair processes such as NHEJ are likely still fully functional in these cells and can effectively "mop up" additional DNA DSB induced by PARP-1 inhibition.

In conclusion this fundamental study of radiation sensitivity in a collection of B-lymphoblastoid cells has revealed potentially important clinical issues. Firstly, in patients with monoallelic mutations in either $B R C A 1$ or BRCA2 are unlikely to experience abnormally high levels of NTT during or after radiotherapy. Secondly we observe a haploinsufficient phenotype in cells with mono-allelic mutations of the $B R C A 1$ gene when radiotherapy is combined with PARP-1 inhibition by Olaparib. While this observation requires further validation with a larger number of cells lines, the data reveal tantalising evidence that the clinical application of PARP inhibitors used concomitantly with radiotherapy may increase the number of individuals that experience radiation induced NTT.

\section{Acknowledgments}

Dr. Emma Bourton was supported by a grant from the Vidal Sassoon Foundation of America. This research was also supported in part by a grant from "The Balls to Cancer" Charity, Coventry, UK

\section{References}

1. Yoshida K, Miki Y (2004) Role of BRCA1 and BRCA2 as regulators of DNA repair, transcription, and cell cycle in response to DNA damage. Cancer Sci 95: 866-871.
2. Venkitaraman AR (2001) Functions of BRCA1 and BRCA2 in the biological response to DNA damage. J Cell Sci 114: 3591-3598.

3. Burma S, Chen BP, Chen DJ (2006) Role of non-homologous end joining (NHEJ) in maintaining genomic integrity. DNA Repair (Amst) 5: 1042-1048.

4. Scott SP, Pandita TK (2006) The cellular control of DNA double-strand breaks. J Cell Biochem 99: 1463-1475.

5. Roy R, Chun J, Powell SN (2012) BRCA1 and BRCA2: different roles in a common pathway of genome protection. Nat Rev Cancer 12: 68-78.

6. Oliver AW, Swift S, Lord CJ, Ashworth A, Pearl LH (2009) Structural basis for recruitment of BRCA2 by PALB2. EMBO Rep 10: 990-996.

7. Kote-Jarai Z, Eeles RA (1999) BRCA1, BRCA2 and their possible function in DNA damage response. Br J Cancer 81: 1099-1102.

8. Lawrence TS, Ten Haken RK, Giaccia A (2008) Principles of Radiation Oncology. In: DeVita VT Jr, Lawrence TS, Rosenberg SA, eds. Cancer: Principles and Practice of Oncology, ( $8^{\text {th }}$ edn). Philadelphia: Lippincott Williams and Wilkins 307-336

9. Cox JD, Stetz J, Pajak TF (1995) Toxicity criteria of the Radiation Therapy Oncology Group (RTOG) and the European Organization for Research and Treatment of Cancer (EORTC) Int J Radiat Oncol Biol Phys 31: 1341-1346.

10. Trotti A, Colevas AD, Setser A, Rusch V, Jaques D, et al. (2003) CTCAE v3.0: development of a comprehensive grading system for the adverse effects of cancer treatment. Semin Radiat Oncol 13: 176-181.

11. Lavin MF, Shiloh $Y$ (1997) The genetic defect in ataxia-telangiectasia. Annu Rev Immunol 15: 177-202.

12. Abbaszadeh F, Clingen PH, Arlett CF, Plowman PN, Bourton EC, et al. (2010) A novel splice variant of the DNA-PKcs gene is associated with clinical and cellular radiosensitivity in a patient with xeroderma pigmentosum. J Med Genet 47: 176-181.

13. Buchholz TA, Wu X, Hussain A, Tucker SL, Mills GB, et al (2002) Evidence of haplotype insufficiency in human cells containing a germline mutation on BRCA1 or BRCA2. International Journal of Cancer 97: 557-561.

14. Leong T, Whitty J, Keilar M, Mifsud S, Ramsay J, et al. (2000) Mutation analysis of BRCA1 and BRCA2 cancer predisposition genes in radiation hypersensitive cancer patients. Int J Radiat Oncol Biol Phys 48: 959-965.

15. Davar D, Beumer JH, Hamieh L, Tawbi H (2012) Role of PARP inhibitors in cancer biology and therapy. Curr Med Chem 19: 3907-3921.

16. McGlynn P, Lloyd RG (2002) Recombinational repair and restart of damaged replication forks. Nat Rev Mol Cell Biol 3: 859-870.

17. Plummer ER (2006) Inhibition of poly(ADP-ribose) polymerase in cancer. Curr Opin Pharmacol 6: 364-368.

18. Mosmann T (1983) Rapid colorimetric assay for cellular growth and survival: application to proliferation and cytotoxicity assays. J Immunol Methods 65: 5563.

19. Green LM, Reade JL, Ware CF (1984) Rapid colorimetric assay for cell viability: application to the quantitation of cytotoxic and growth inhibitory lymphokines. $J$ Immunol Methods 70: 257-268.

20. Weil MK, Chen AP (2011) PARP inhibitor treatment in ovarian and breast cancer. Curr Probl Cancer 35: 7-50.
This article was originally published in a special issue, Cancer Genetics handled by Editor(s). Dr. Ahmed M Malki, Alexandria University, Egypt
Citation: Bourton EC, Foster HA, Plowman PN, Harvey AJ, Parris CN (2013) Hypersensitivity of BRCA1 Heterozygote Lymphoblastoid Cells to Gamma Radiation and PARP Inhibitors. J Genet Syndr Gene Ther 4: 146. doi:10.4172/2157-7412.1000146 